\title{
CORRECTION
}

\section{Correction to: Dual Antiplatelet Therapy Duration in Medically Managed Acute Coronary Syndrome Patients: Sub-Analysis of the OPT-CAD Study}

Sicong Ma $\cdot$ Zaixin Jiang $\cdot$ Miaohan Qiu $\cdot$ Zhiguo Li $\cdot$ Liya Bian $\cdot$

Yi Li $\cdot$ Yaling Han (1)

Published online: June 9, 2020

(c) Springer Healthcare Ltd., part of Springer Nature 2020

Correction to: Adv Ther

https://doi.org/10.1007/s12325-020-01376-0

In the original article, the incorrect funding statement has been published. The correct funding statement is "This study is supported by the national key R\&D project (contract no. 2016YFC1301303, 2016YFC1301300) and an investigator-initiated grant by AstraZenca Co. Ltd. No funding was received for the journals rapid service fee".

The original article can be found online at https://doi. org/10.1007/s12325-020-01376-0.

S. Ma · Z. Jiang · M. Qiu · Z. Li · L. Bian · Y. Li ·

Y. Han $(\square)$

The Department of Cardiology, General Hospital of

Northern Theater Command, Shenyang, Liaoning

Province, China

e-mail: han1320288258@qq.com

S. Ma

The Department of Cardiology, Xijing Hospital, Air Force Military Medical University, Xi'an, Shanxi

Province, China

Z. Jiang

Navy Medical University, Shanghai, China 